CERN-TH/96-229

hep-th/9608171

\title{
CONDENSATION OF p-BRANES AND GENERALIZED HIGGS/CONFINEMENT DUALITY*
}

\author{
FERNANDO QUEVEDO \\ Theory Division, CERN \\ CH-1211 Geneva 23, Switzerland \\ CARLO A. TRUGENBERGER \\ Department of Theoretical Physics, University of Geneva \\ CH-1211 Geneva 4, Switzerland
}

\begin{abstract}
We review our recent work on the low-energy actions and the realizations of strongweak coupling dualities in non-perturbative phases of compact antisymmetric tensor field theories due to p-brane condensation. As examples we derive and discuss the confining string and confining membrane actions obtained from compact vector and tensor theories in $4 \mathrm{D}$. We also mention the relevance of our results for the description of the Hagedorn phase transition of finite temperature strings.
\end{abstract}

Dualities play an ever increasing role in modern high-energy physics. ${ }^{1}$ Accordingly, both field theory and string dualities are the subject of current intensive investigations.

Field theory dualities can be roughly divided into Abelian and non-Abelian dualities, the former being much better understood since they can be derived from an action principle. The paramount examples of this strong-weak coupling Abelian dualities are given by a massless scalar field theory in $2 \mathrm{D}$ and by $4 \mathrm{D}$ sourceless QED.

The natural generalization of these two examples is given by generic antisymmetric tensor field theories ${ }^{2,3}$ with (Euclidean) action

$$
S=\int \frac{1}{e^{2}} d \phi_{h-1} \wedge * d \phi_{h-1},
$$

where $\phi_{h-1}$ is an $(h-1)$-form in $D=d+1$ space-time dimensions and $e^{2}$ is a dimensionless coupling constant. The $U(1)$ gauge invariance under transformations

\footnotetext{
* To appear in the proceedings of the 1996 summer Telluride workshop.
} 
$\phi_{h-1} \rightarrow \phi_{h-1}+d \lambda_{h-2}$ implies that (11) describes $\left(\begin{array}{l}d-1 \\ h-1\end{array}\right)$ massless degrees of freedom.

The tensor $\phi_{h-1}$ couples naturally to a closed $(h-2)$-dimensional extended object: in modern parlance a $(h-2)$-brane:

$$
\begin{aligned}
S & \rightarrow S+i \tilde{q} \int \phi_{h-1} \wedge * \tilde{j}_{h-1}, \\
\tilde{j}_{h-1}^{\mu_{1} \ldots \mu_{h-1}} & =\int \delta^{d+1}(x-\tilde{y}(\tilde{\sigma})) d \tilde{y}^{\mu_{1}} \wedge \ldots \wedge d \tilde{y}^{\mu_{h-1}},
\end{aligned}
$$

where $\tilde{q}$ is a coupling of canonical dimension $(2 h-d-1) / 2$ and $\tilde{y}(\tilde{\sigma})$ parametrizes the closed (or infinitely extended) world-hypersurface of the $(h-2)$-brane. Accordingly, (11) can be viewed as an Abelian gauge theory for closed $(h-2)$-branes.

The model dual to (1D) is formulated in terms of a $(d-h)$-form $\tilde{\phi}_{d-h}$ defined by $d \tilde{\phi}_{d-h}=* d \phi_{h-1}$ and has the action

$$
\tilde{S}=\int \frac{e^{2}}{4} d \tilde{\phi}_{d-h} \wedge * d \tilde{\phi}_{d-h}
$$

Note that the coupling constant is reversed so that a strong-coupling regime of (1) corresponds to a weak-coupling regime of (4) and viceversa. This model can be viewed as a gauge theory for closed (or infinitely extended) $(d-h-1)$-branes:

$$
\begin{aligned}
S & \rightarrow S+i q \int \tilde{\phi}_{d-h} \wedge * j_{d-h} \\
j_{d-h}^{\mu_{1} \ldots \mu_{d-h}} & =\int \delta^{d+1}(x-y(\sigma)) d y^{\mu_{1}} \wedge \ldots \wedge d y^{\mu_{d-h}},
\end{aligned}
$$

where the coupling $q$ has dimension $(d-2 h+1) / 2$.

Higher-rank antisymmetric tensors appear naturally in supersymmetric field theories and play an ubiquitous role in the field theory low-energy limits of various string theories. ${ }^{4}$ The above duality transformation plays a key role in the realization of strong-weak coupling dualities among string theories. ${ }^{5}$

The relation between (11) and (4) is the simplest example of the realization of an Abelian duality in field theory. In the following we wish to discuss a simple, yet highly non-trivial generalization which is still tractable in general. This is obtained by considering compact antisymmetric field theories, which are the generalizations of Polyakov's compact QED ${ }^{6}$ to higher-rank tensor theories. ${ }^{7}$

In compact antisymmetric field theories $p$-branes appear also as topological defects of the original theory. For the model (11) these can be viewed e.g. as closed $(d-h)$-dimensional singularities excluded from the definition domain of the (Euclidean) model. This is then considered as a low-energy effective field theory valid only 'outside' these singularities, which represent the world-hypersurfaces of $(d-h-1)$-branes.

The presence of these singularities induces non-trivial homology cycles for the antisymmetric tensor fields. Let us cut one of these singularities with an $(h+1)$ dimensional hyperplane $\Sigma_{h+1}$ and let us choose a sphere $S_{h}$ on $\Sigma_{h+1}$ around one 
of the two intersection points with the $(d-h)$-dimensional singularity. The topological quantum number is then given by $\int_{S_{h}} d \phi_{h-1}$. Note that in this formulation instantons appear as (-1)-branes.

As always for effective field theories, a proper definition of the model requires an ultraviolet cutoff. For vector theories one can obtain the compact $U(1)$ group by spontaneous symmetry breaking of a compact non-Abelian group. In this case the cutoff is provided by the mass of the broken gauge fields. For higher-rank tensors this interpetation is no more possible. Keeping in mind that antisymmetric tensors appear in the low-energy limit of string theories one could think of the cutoff as provided by massive string modes. In such models, however, the low-energy theory contains typically additional massless fields, as the graviton and the dilaton. The gravitational sector, in particular, shields the singularities by event horizons, leading to finite masses of solitonic $p$-branes already in the low-energy theory. ${ }^{8}$ In the following we shall not include the graviton and the dilaton but deal rather only with compact antisymmetric tensors. When needed one can always resort to a lattice regularization, a procedure that works for any rank of the tensors.

We shall consider both the original tensor $\phi_{h-1}$ and its dual $\tilde{\phi}_{d-h}$ to be compact, so that we have two types of topological defects: $(d-h-1)$-branes for $\phi_{h-1}$ and $(h-2)$-branes for $\tilde{\phi}_{d-h}$. In this case the dual actions (11) and (4) have to be modified in order to take into account explicitly the topological defects. The new pair of dual actions is given by

$$
\begin{aligned}
& S=\int \frac{1}{e^{2}}\left(d \phi_{h-1}-q V_{h}\right) \wedge *\left(d \phi_{h-1}-q V_{h}\right)+i \tilde{q} \phi_{h-1} \wedge * \tilde{j}_{h-1} \\
& \tilde{S}=\int \frac{e^{2}}{4}\left(d \tilde{\phi}_{d-h}-\tilde{q} \tilde{V}_{d+1-h}\right) \wedge *\left(d \tilde{\phi}_{d-h}-\tilde{q} \tilde{V}_{d+1-h}\right)+i q \tilde{\phi}_{d-h} \wedge * j_{d-h},(8)
\end{aligned}
$$

where the (singular) forms $V$ and $j$ describing the topological defects are defined as follows:

$$
\begin{aligned}
* V_{h} & =T_{d-h+1}, & & j_{d-h}=\delta T_{d-h+1}, \\
* \tilde{V}_{d+1-h} & =-(-1)^{h(d+1-h)} \tilde{T}_{h}, & & \tilde{j}_{h-1}=\delta \tilde{T}_{h} .
\end{aligned}
$$

Here

$$
\begin{aligned}
T_{d-h+1}^{\mu_{1} \ldots \mu_{d-h+1}} & =\int \delta^{d+1}(x-y(\sigma)) d y^{\mu_{1}} \wedge \ldots \wedge d y^{\mu_{d-h+1}}, \\
\tilde{T}_{h}^{\mu_{1} \ldots \mu_{h}} & =\int \delta^{d+1}(x-\tilde{y}(\tilde{\sigma})) d \tilde{y}^{\mu_{1}} \wedge \ldots \wedge d \tilde{y}^{\mu_{h}} .
\end{aligned}
$$

Here $T_{d-h+1}$ and $\tilde{T}_{h}$ are the volume forms for open hypervolumes $y(\sigma)$ and $\tilde{y}(\tilde{\sigma})$ which describe the generalization to higher-dimensional topological defects of the familiar Dirac string connecting a monopole-antimonopole pair. The boundaries of these hypervolumes, described by the (tensor) currents $j_{d-h}$ and $\tilde{j}_{h-1}$, are the world-hypersurfaces of the topological defects. Note that these are now dynamical objects which have to be traced over in the partition functions corresponding to the 
above actions. The couplings $q$ and $\tilde{q}$ represent the 'charge' units of the $(d-h-1)$ branes and $(h-2)$-branes respectively: they are the generalization of the familiar magnetic and electric charge units $g$ and $e$. Establishing duality of the above pair of actions requires a generalized Dirac quantization condition

$$
q \tilde{q}=2 \pi p, \quad p \in Z .
$$

The solitonic $(d-h-1)$-branes of the compact version of (11) couple as 'Noether currents' to the dual tensor field $\tilde{\phi}_{d-h}$, as in (6), and viceversa.

Topological defects can condense, leading to drastic modifications of the infrared behaviour of the original theory. ${ }^{6}$ When studying such effects two separated questions have to be answered. The first regards the condensation dynamics and can be addressed best with a lattice regularization: the condensation mechanism is rather well established ${ }^{6,7,9}$ for $h=d((-1)$-branes $=$ instantons $)$ and for $h=d-1(0$ branes $=$ monopoles), although there are also partial results on the condensation of higher-dimensional branes. ${ }^{10}$ The second question, which we would like to address here, regards the nature of the new phase with a continuous distribution of topological defects and the low-energy effective action describing it.

This question has been addressed nearly twenty years ago by Julia and Toulouse ${ }^{11}$ in the context of ordered solid state media. In a nutshell, their idea is that the condensation of topological defects gives rise to new low-energy (hydrodynamical) modes representing the long-wavelength fluctuations about the homogeneous distribution of topological defects. While in the framework of ordered solid state media this idea did not bring very far due to non-linearities and the need of introducing dissipation terms, it is perfectly apt to study compact antisymmetric tensor theories, where it naturally leads also to the effective action for the new phase. ${ }^{12}$

Let us concentrate first on the original model (11). In our framework the idea of Julia and Toulouse can be implemented simply by promoting the singular form $V_{h}$ in (10) to a new tensor field $\omega_{h}$. Since the conserved (tensor) current describing the fluctuations about the homogenoeus distribution of topological defects is $j_{d-h} \propto$ $* d \omega_{h}$, the new degrees of freedom are associated with the gauge invariant part of $\omega_{h}$.

Condensation of $(d-h-1)$-branes generates a new scale $\Lambda$. This can be taken as $\Lambda \propto \rho^{1 / h+1}$, where $\rho$ is the average density of intersection points of the $(d-h)$-dimensional world-hypersurfaces of the condensed branes with any $(h+1)$ dimensional hyperplane in the Euclidean space-time.

The three requirements on the effective action for the dense phase are gauge invariance under transformations $\omega_{h} \rightarrow \omega_{h}+d \psi_{h-1}$, relativistic invariance and recovering the original model in the limit $\Lambda \rightarrow 0$. Up to two derivatives in the new field we are thus led to the action

$$
S_{d-h-1}=\int \frac{1}{\Lambda^{2}} d \omega_{h} \wedge * d \omega_{h}+\frac{1}{e^{2}}\left(\omega_{h}-d \phi_{h-1}\right) \wedge *\left(\omega_{h}-d \phi_{h-1}\right),
$$

where gauge invariance is realized by accompanying transformations $\omega_{h} \rightarrow \omega_{h}+$ $d \psi_{h-1}$ with transformations $\phi_{h-1} \rightarrow \phi_{h-1}+\psi_{h-1}$. 
This gauge invariance must be gauge fixed. As always for Abelian theories, this means that one can drop the integration over $\phi_{h-1}$ after reabsorbing $d \phi_{h-1}$ into $\omega_{h}$, so that the action describes $\left(\begin{array}{l}d \\ h\end{array}\right)$ physical degrees of freedom of mass $m=\Lambda / e$. This mechanism, which we called the Julia-Toulouse mechanism, is the exact opposite of the familiar Higgs-Stückelberg mechanism: the new degrees of freedom, generated by the condensation of topological defects, 'eat' the original degrees of freedom thereby acquiring a mass.

In order to establish the nature of this phase we note that the original coupling (3) to closed $(h-2)$-branes has to be promoted to a new coupling

$$
S_{d-h-1} \rightarrow S_{d-h-1}+i \tilde{q} \int \omega_{h} \wedge * \tilde{T}_{h}
$$

where $\tilde{T}_{h}$ describes an open hypervolume bounded by the world-hypersurface of the original $(h-2)$-brane. Since $\omega_{h}$ is a massive field, the induced action for $\tilde{T}_{h}$ reduces in the infrared to

$$
S_{\text {ind }}=e^{2} \tilde{q}^{2} \int \tilde{T}_{h} \wedge * \tilde{T}_{h}+\ldots,
$$

which represents a generalized Wilson 'hypervolume law'. This shows that the Julia-Toulouse mechanism describes the transition to a confinement phase for the $(h-2)$-branes.

At this point we can ask what is the strong-weak coupling dual formulation of (14). This turns out to be

$$
\tilde{S}_{d-h-1}=\int \frac{e^{2}}{4} d \tilde{\phi}_{d-h} \wedge * d \tilde{\phi}_{d-h}+\frac{\Lambda^{2}}{4}\left(\tilde{\phi}_{d-h}-d \tilde{\omega}_{d-h-1}\right) \wedge *\left(\tilde{\phi}_{d-h}-d \tilde{\omega}_{d-h-1}\right),
$$

where $d \phi_{h-1}=* d \tilde{\phi}_{d-h}$ and $d \omega_{h}=* d \tilde{\omega}_{d-h-1}$. In this formulation we recognize a generalized version of the Higgs-Stückelberg mechanism: the original field $\tilde{\phi}_{d-h}$ 'eats' the new field $\tilde{\omega}_{d-h-1}$ due to the condensation of topological defects and acquires thereby a mass $m=\Lambda / e$. We have thus shown that the Julia-Toulouse mechanism is the strong-weak coupling dual of a generalized Higgs-Stückelberg mechanism. Correspondingly, the confinement phase for $\phi_{h-1}$ is equivalent to a Higgs phase for its dual $\tilde{\phi}_{d-h}$. This provides an explicit realization of the old ideas of 't Hooft and Mandelstam, along with a generalization to generic antisymmetric tensor field theories. It also provides a clue to non-perturbative realizations of strong-weak coupling dualities and shows that various antisymmetric tensor field theories are connected by the condensation of $p$-branes. ${ }^{13}$

Up to now we have considered only the condensation of $(d-h-1)$-branes. Naturally, corresponding results are obtained due to the condensation of the dual $(h-2)$-branes. In this case one obtains a Higgs phase for $\phi_{h-1}$, or, equivalently, a confinement phase for the dual $\tilde{\phi}_{d-h}$.

The most interesting examples of this non-perturbative phases are obtained for $h=(d+1) / 2$. In these cases both types of topological defects have the same dimension and the Higgs and confinement phases are described by tensors of the 
same rank. We thus expect a phase diagram which is symmetric around the selfdual point $e^{2}=2$. In $D=2,4$ this is triggered by instantons and monopoles, respectively, and is realized as follows: there is a Higgs phase for $\phi_{h-1}$ at weak coupling and a confinement phase for $\phi_{h-1}$ at strong coupling with the possibility of an intermediate self-dual Coulomb (massless) phase for $p$ in (13) larger than a critical value. ${ }^{9}$ The next interesting case regards string condensation in $D=6$. There is evidence that the same structure is realized. ${ }^{7}$

To conclude this brief review we shall discuss two concrete examples which are relevant for two different types of string theories. The first example is compact $Q E D^{6}$ in $(3+1)$ dimensions, with (Euclidean) action

$$
S=\int d^{4} x \frac{1}{4 e^{2}} F_{\mu \nu} F_{\mu \nu}, \quad F_{\mu \nu} \equiv \partial_{\mu} A_{\nu}-\partial_{\nu} A_{\mu} .
$$

This is a case for which $h=(d+1) / 2$ : both types of topological defects are 0-branes, i.e. point particles. The topological defects of (18) are magnetic monopoles, while their dual topological defects are electric charges, which have the standard Noether coupling to $A_{\mu}$.

The condensation of electric charges leads to the familiar Higgs phase with effective low-energy action

$$
S_{H}=\int d^{4} x \frac{1}{4 e^{2}} F_{\mu \nu} F_{\mu \nu}+\frac{\tilde{\Lambda}^{2}}{2} A_{\mu} A_{\mu},
$$

describing a massive vector of mass $m_{H}=e \tilde{\Lambda}$. In this phase the magnetic monopoles are confined, which is tantamount to the Meissner effect. The condensation of magnetic monopoles, instead, leads to a confinement phase with effective low-energy action given by

$$
S_{C}=\int d^{4} x \frac{1}{12 \Lambda^{2}} \partial_{[\mu} B_{\nu \alpha]} \partial_{[\mu} B_{\nu \alpha]}+\frac{1}{4 e^{2}} B_{\mu \nu} B_{\mu \nu},
$$

describing a massive two-index tensor of mass $m_{C}=\Lambda / e$. In this phase electric charges are confined.

Actually the induced action

$$
\mathrm{e}^{-S_{\text {ind }}(\Sigma)}=\frac{1}{Z\left(B_{\mu \nu}\right)} \int \mathcal{D} B_{\mu \nu} \mathrm{e}^{-S_{C}\left(B_{\mu \nu}\right)+i \int_{\Sigma} d \sigma_{\mu \nu} B_{\mu \nu}}
$$

defines the (low-energy) action for the (Abelian) confining string with world-sheet $\Sigma$, a result recently pointed out also by Polyakov. ${ }^{14}$

The second example we shall discuss is the puzzle of the axion mass in $4 \mathrm{D}$ string models. In its simplest formulation, the axion is a pseudoscalar with a 'Noether' coupling to the QCD instantons: ${ }^{15}$

$$
S=\int d^{4} x \frac{1}{2} \partial_{\mu} a \partial_{\mu} a+i a \frac{1}{16 \pi^{2} f} \operatorname{Tr} F_{\mu \nu} F_{\mu \nu}^{*} .
$$


Instantons are always in a plasma phase for $D>2$. As is well known ${ }^{15}$ these instantons generate a potential $V(a)$ for the axion field. By considering only small instantons this is given by $V(a)=\Lambda_{Q C D}^{4}(1-\cos (a / f))$ which implies an axion mass $m_{a}=\Lambda_{Q C D}^{2} / f$. In our terminology this would be a 'Higgs phase' for the axion.

In $4 \mathrm{D}$ string models, however, the axion appears in its dual formulation, given in terms of the Kalb-Ramond ${ }^{2}$ two-index tensor $B_{\mu \nu}$ :

$$
\tilde{S}=\int d^{4} x \frac{1}{f^{2}}\left(\partial_{[\mu} B_{\nu \alpha]}-K_{\mu \nu \alpha}\right)\left(\partial_{[\mu} B_{\nu \alpha]}-K_{\mu \nu \alpha}\right),
$$

where $K_{\mu \nu \alpha}$ is the Chern-Simons three-form defined by

$$
\epsilon_{\mu \nu \alpha \beta} \partial_{\mu} K_{\nu \alpha \beta}=-\frac{1}{16 \pi^{2}} \operatorname{Tr} F_{\mu \nu} F_{\mu \nu}^{*} .
$$

Up until recently, the origin and description of the axion mass in this formulation were a puzzle. In our formalism, however, the solution of this puzzle is very simple. Indeed, in the dual formulation small instantons play the role of the topological defects of a compact $B_{\mu \nu}$ field, the so-called axionic instantons. ${ }^{6,16}$ Since these are always in a plasma phase we obtain the low-energy effective action

$$
\tilde{S}_{-1}=\int d^{4} x \frac{1}{4 \Lambda_{Q C D}^{4}} \partial_{[\mu} H_{\nu \alpha \beta]} \partial_{[\mu} H_{\nu \alpha \beta]}+\frac{1}{f^{2}} H_{\mu \nu \alpha} H_{\mu \nu \alpha},
$$

formulated in terms of a massive three-index tensor of mass $m_{H}=\Lambda_{Q C D}^{2} / f$. This corresponds thus to a string confinement phase. That in 4D strings are confined and turn to membranes has been, to our knowledge, first proposed by Polyakov ${ }^{17}$ and subsequently rediscovered by S.-J. Rey. ${ }^{16}$ Our result provides an explicit realization of this mechanism and the low-energy effective action for this confinement phase. Actually, with a construction analogous to (21), the induced action obtained by coupling (25) to the world-volume of the membrane defines the action for the 'confining membrane'.

This example is particularly important since the result (25) can be obtained (it was actually first obtained this way) from the supersymmetric version of (23) due to compactification of the heterotic string. ${ }^{18}$ In this case instantons drive gaugino condensation which implies the appearance of a massive three-index tensor in the spectrum. This result can be considered as a supersymmetric version of the confining membrane. Moreover, it suggests the existence of a generic supersymmetric version of the Julia-Toulouse mechanism, which would describe the transition to non-perturbative phases of string theories. Since the dilaton is always in the same supermultiplet with the antisymmetric tensor, either it also acquires a mass or supersymmetry is broken in these phases. At least one of the outstanding problems of string theory could be solved this way.

Let us finish with a description of the $2 \mathrm{D}$ scalar field $X$ living on a circle of radius $R$, relevant for toroidal string compactifications. Since $h=(d+1) / 2=1$, the 
corresponding topological defects are instantons and dual instantons. The action in the Coulomb phase is $4 \pi \alpha^{\prime} S=\int d^{2} z \partial_{\mu} X \partial^{\mu} X+\cdots$ with periodicity $X \equiv X+2 \pi R$. The dual action is identical in terms of the dual coordinate $\tilde{X}$ with periodicity $\tilde{X} \equiv \tilde{X}+2 \pi p \alpha^{\prime} / R$, with $p$ the Dirac quantum of equation (13). Following our prescription, the confinement phase is described by a massive vector dual to a massive scalar $4 \pi \alpha^{\prime} S_{C}=\int d^{2} z\left\{\partial_{\mu} \tilde{X} \partial^{\mu} \tilde{X}-\Lambda^{2} R^{2} / \alpha^{\prime 2} \tilde{X}^{2}+\cdots\right\}$, with a similar expression for the Higgs phase (exchanging $X \leftrightarrow \tilde{X}, R \leftrightarrow p \alpha^{\prime} / R, \Lambda \leftrightarrow \tilde{\Lambda}$ ) and thus showing a Higgs/confinement duality. As mentioned above, the phase diagram is symmetric around the selfdual point $R^{2}=p \alpha^{\prime}$. This was thoroughly studied in reference $[9]$ in terms of the 'mass formula'.

$$
\alpha^{\prime} M^{2}=p^{2} n^{2} \frac{\alpha^{\prime}}{R^{2}}+m^{2} \frac{R^{2}}{\alpha^{\prime}}+2\left(N_{L}+N_{R}-2\right) .
$$

If $X$ is a string coordinate, this is the standard mass formula (for $p=1$ ) with $n$ the quantized momentum, $m$ the winding number and $N_{L, R}$ the left and right moving oscillator numbers. It can be seen that the $2 \mathrm{D}$ instantons interpolate among different windings and therefore the dual instantons interpolate among different momenta. Using the previous equation, Cardy and Rabinovici extracted the phase diagram of this system [9]. A way to see this is to find the points in $R$-space where tachyons appear. We can see that for $n=N_{L, R}=0, m= \pm 1$ there is a tachyon for $R^{2}<4 \alpha^{\prime}$ whereas for $m=N_{L, R}=0, n= \pm 1$ there is a tachyon for $R^{2}>p^{2} \alpha^{\prime} / 4$. For $p>4$ there is a range $4 \alpha^{\prime}<R^{2}<p^{2} \alpha^{\prime} / 4$ without an extra tachyon and in that interval the (selfdual) Coulomb phase is realized whereas the Higgs phase exists for larger values of $R^{2}$, dual to the confinement phase at smaller values of $R^{2}$. For $p<4$ there are only Higgs and confinement phases emanating at the selfdual point. This is the case for modular invariant bosonic strings with $p=1$. This shows explicitly how $T$ duality is preserved in the presence of nonperturbative effects once both instantons and dual instantons are taken into account.

For perturbative supersymmetric strings there are no tachyons in the spectrum for any value of $R$ and then only the Coulomb phase is realized. However, at finite temperature, the situation is again interesting. In this case, $X$ is the time coordinate and the temperature is the inverse period $T=1 / 2 \pi R$. The mass formula is (for $p=1)[19]$ :

$$
\alpha^{\prime} M^{2}=n^{2} \frac{\alpha^{\prime}}{R^{2}}+m^{2} \frac{R^{2}}{\alpha^{\prime}}+2\left(N_{L}+N_{R}-\frac{3}{2}\right),
$$

with the level matching condition: $m n=\left(N_{R}-N_{L}+1 / 2\right)$. The difference with the purely bosonic case relies on the fact that $n$ can be half-integer. Actually the states with $m= \pm 1, n=m / 2, N_{L, R}=0$ are tachyonic in the range $1+1 / \sqrt{2}>R / \sqrt{\alpha^{\prime}}>$ $1-1 / \sqrt{2}$. Since the tachyonic states carry both momentum and winding, the corresponding instantons will be 'dyonic instantons'. Therefore there is a Coulomb phase at temperatures below the Hagedorn temperature $T_{H} \equiv(\sqrt{2}-1) / \sqrt{2 \alpha^{\prime}} \pi$, where the condensation of 'mdyons' induce a phase transition. This (selfdual) phase 
finishes at the dual Hagedorn temperature $\tilde{T}_{H} \equiv(\sqrt{2}+1) / \sqrt{2 \alpha^{\prime}} \pi$ where the tachyon disappears and the dual Coulomb phase starts.

The physical picture we get is the following: for temperatures $T<T_{H}$ the system is in a Coulomb phase described in terms of a worldsheet action with compact euclidean time defining temperature. At temperatures $T_{H}<T<\tilde{T}_{H}$ there is the 'dyon condensate' phase, described by a massive vector field dual to a 'massive time coordinate'. Since standard euclidean time is no longer well defined in this phase, $T$ may no longer be the physical temperature. Instead, an effective temperature $T_{\text {eff }}$ depending on both $T$ and the mass parameter $\Lambda$ will have to be defined up to the selfdual point. Beyond that point, the whole picture is repeated but now in terms of the dual variables. This might solve the problem of how to make compatible temperature duality with the fact that the partition function should be a monotonic function of temperature [19]. Therefore, temperature duality may also be an exact symmetry of string theory. This could have very interesting cosmological implications. It may also be interesting to find a supersymmetric generalization of our approach to compare with the results of ref. [20] where a target space formulation was used.

In summary we have seen how, in our approach, duality is always a good nonperturbative symmetry once all the relevant topological defects are included. The generalized Higgs and confinement phases we have described may actually be present in nonperturbative string theory where $p$-branes are playing a fundamental role. We may actually be in a $p$-brane confining phase where the dilaton field is no longer massless.

\section{Acknowledgements}

C. A. Trugenberger is supported by a Profil 2 fellowship of the Swiss National Science Foundation.

\section{References}

1. For a recent review see: D. Olive, hep-th/9508089.

2. V. I. Ogievetsky and I. V. Polubarinov, Sov. J. Nucl. Phys. 4 (1967) 156; M. Kalb and P. Ramond, Phys. Rev. D9 (1974) 2273.

3. C. Teitelboim, Phys. Lett. 167 B (1986) 63, Phys. Lett. $167 B$ (1986) 69; R. I. Nepomechie Phys. Rev. D31 (1984) 1921.

4. M. Green, J. Schwarz and E. Witten, Superstring Theory (Cambridge University Press, Cambridge, 1987).

5. For a recent review see: J.H. Schwarz, hep-th/9607201.

6. A. Polyakov, Gauge Fields and Strings (Harvood Academic Publishers, Chur, 1987).

7. R. Savit, Rev. Mod. Phys. 52 (1980) 453; P. Orland Nucl. Phys. B205[FS5] (1982) 107

8. For a review see: M. J. Duff, R. Khuri and J.Lu, Phys. Rep. 259 (1995) 213.

9. J. L. Cardy and E. Rabinovici, Nucl. Phys. B205[FS5] (1982) 1.

10. D. Foerster, Phys. Lett. $77 B$ (1978) 211; S.-J. Rey, Phys. Rev. D40 (1989) 3396.

11. B. Julia and G. Toulouse, J. Physique Lett. 40 (1979) 396.

12. F. Quevedo and C. A. Trugenberger, hep-th/9604196. 
13. See also: M. C. Diamantini, hep-th/9607090.

14. A. Polyakov, hep-th/9607049.

15. For a review see: R. D. Peccei, in CP Violation, ed. C. Jarlskog (World Scientific, Singapore, 1989).

16. S.-J. Rey, Phys. Rev. D43 (1991) 526.

17. A. Polyakov, Physica Scripta T15 (1987) 191.

18. C. P. Burgess, J.-P. Derendinger, F. Quevedo and M. Quiros, Phys. Lett. B348 (1995) 428.

19. K.H. O'Brien and C.I. Tan, Phys. Rev. D36 (1987) 1184; E. Alvarez and M.A.R. Osorio, Nucl. Phys. B304 (1988) 327; J. Atick and E. Witten, Nucl. Phys. B310, (1988) 291.

20. I. Antoniadis and C. Kounnas, Phys. Lett. B261 (1991) 369. 